\title{
Análise do Comportamento de Recidivas x Reações Hansênicas em Alagoas
}

\author{
Analysis of the Behavior of Relapse x Leprosy Reactions in Alagoas
}

Análisis del Comportamiento de las Recurrencias x Reacciones de Lepra en Alagoas

\author{
Clodis Maria Tavares ${ }^{1}$ \\ Simone Lira Targino ${ }^{2}$ \\ Vanessa Almeida do Nascimento ${ }^{3}$ \\ Nataly Mayara Cavalcante Gomes ${ }^{4}$ \\ Pétalla Morganna Figueiredo Pessoa de Barros ${ }^{5}$ \\ Fernanda da Silva Goes ${ }^{6}$
}

\section{Resumo}

Objetivo: Analisar o comportamento dos casos de recidiva de hanseníase de residentes em Alagoas. Método: Foi realizado um estudo descritivo, retrospectivo, com abordagem quantitativa. Resultados: Após cruzamento de 76 fichas de notificação de hanseníase com modo de entrada de recidiva na segunda notificação comparando com o modo de entrada como caso novo da primeira ficha no período estudado, os dados denotaram que 37 casos (49\%), eram recidivas reais, segundo critérios estabelecidos e 39 (51\%) de falsas recidivas, por não cumprir o número de anos de alta por cura (5 anos), na realidade estudos mostram que são casos de Reações Hansênicas. Observa-se que as entradas por recidiva se deram em 24 (30\%) em

${ }^{1}$ Enfermeira, Docente Doutora da Escola de Enfermagem e Farmácia da Universidade Federal de Alagoas. Autora correspondente: Campus A. C. Simões. Av. Lourival Melo Mota, s/n. Tabuleiro dos Martins. 57072-900. Maceió, Al, Brasil. E-mail: clodistavares@yahoo.com.br

${ }^{2}$ Funcionária Técnica da Diretoria de Vigilância Epidemiológica - DIVEP da Secretaria Estadual de Saúde de Alagoas.

${ }^{3}$ Enfermeira da Secretaria Municipal de Maceió.

${ }^{4}$ Acadêmica de Enfermagem da Universidade Federal de Alagoas.

${ }^{5}$ Enfermeira filiada ao Conselho Regional de Enfermagem do Estado de Alagoas.

${ }^{6}$ Enfermeira filiada ao Conselho Regional de Enfermagem do Estado de Alagoas.

Recebido: Abri/2017 - Aceito: Jul/2017. 
Unidades básicas e $55 \quad(70 \%)$ em Unidades de Referência. Conclusão: Segundo dados de Alagoas no período selecionado, as recidivas apresentaram um alto índice que corresponde a 5,4\% ao ano. Portanto inferir-se que ainda existem dúvidas por parte dos profissionais de saúde em realizar esse diagnóstico e que nem sempre encaminham esses usuários as Unidades de Referência Municipal e/ou Estadual.

\section{Descritores: Recidiva; Hanseníase;}

Autoimune.

\section{Abstract}

Objective: of this study was to analyze the behavior of leprosy relapse cases of residents in Alagoas. Methods: used was a descriptive, retrospective study with a quantitative approach. Results: After crossing 76 leprosy notification forms with relapse entry mode in the second notification comparing with the entry mode as a new case of the first record in the period studied, the data indicated that 37 cases (49\%) were real relapses, according to Established criteria and 39 (51\%) of false recurrences, for failing to meet the number of years of discharge for cure (5 years), in fact studies show that they are cases of Hansenic Reactions. It is observed that the relapse entries occurred in 24 (30\%) in Basic Units and 55 (70\%) in Reference Units. Conclusion: according to data from Alagoas in the selected period, recurrences presented a high index corresponding to $5.4 \%$ per year. Therefore, it is inferred that there are still doubts on the part of the health professionals in making this diagnosis and that they do not always refer these users to the Municipal and / or State Reference Units.

Descriptors: Recurrence; Leprosy; Autoimmunity.

\section{Resumen}

Objetivo: Analizar el comportamiento de los casos de lepra residentes de la recaída en Alagoas. Métodos: Estudio retrospectivo descriptivo con un enfoque cuantitativo. Resultados: Después de pasar 76 formularios de notificación de lepra con modo de entrada recurrente en la segunda notificación comparando el modo de entrada como un nuevo caso de que el primer registro en el período de estudio, que se denota datos de 37 casos (49\%) eran reales segunda recurrencia criterios y 39 (51\%) de los falsos 
recaídas establecida, por no cumplir con el número de años de curación alta (cinco años), en los estudios hechos demuestran que son casos de reacciones de lepra. Se observa que las entradas para la recaída se ha producido en 24 (30\%) en unidades básicas y 55 (70\%) en unidades de referencia. Conclusión: Según los datos de Alagoas, en el periodo actual, las recaídas mostró un alto nivel correspondiente al 5,4\% por año. Por lo tanto se deduce que todavía hay dudas por parte de los profesionales de la salud para realizar este diagnóstico, $y$ no siempre se refieren a estos usuarios de las unidades y / o estado de Referencia Municipal.

\section{Descriptores: Recurrencia; Lepra;}

\section{Autoinmunidad.}

\section{Introdução}

A hanseníase no Brasil é uma doença endêmica e um grave problema de saúde pública. De acordo com a Organização Mundial de Saúde, 102 países e territórios reportaram casos de hanseníase durante o ano de 2014. "Neste ano, o Brasil ocupou a segunda posição em número de casos novos de hanseníase, $\quad$ com 31.064, correspondendo a $14,5 \%$. A primeira posição está com a Índia, com 125.785 casos novos, o que corresponde a 58,8\% do total" (1).

Trata-se de uma doença crônica de origem infecciosa, cujo agente etiológico é o Mycobacterium leprae, que é transmitido através das vias aéreas superiores, quando um indivíduo susceptível tem um contato prolongado com um portador multibacilar que não está em tratamento, sendo que esse microorganismo tem uma capacidade de infectar altíssimas, mas uma baixa patogenicidade $^{(2)}$.

O tratamento da Hanseníase iniciou-se no final de 1940 com a implementação da sulfona no tratamento da doença. A Poliquimioterapia (PQT) foi recomendada pela OMS em 1982 e no Brasil foi implantada gradativamente a partir de 1991, o que possibilitou uma redução de até $75 \%$ na taxa de prevalência desta doença ${ }^{(3)}$.

Apesar da eficácia do tratamento preconizado, surgiram evidências da possível resistência medicamentosa aos quimioterápicos existentes, resultado este, ligado ao uso irregular do mencionado esquema terapêutico, levando à baixa adesão ao tratamento e à possibilidade da ocorrência de recidiva e consequentemente 
permanência da fonte de infecção na comunidade $^{(3)}$.

Os casos de recidiva em hanseníase são raros em pessoas tratadas regularmente, com os esquemas poliquimioterápicos preconizados. Geralmente, ocorrem em período superior a cinco anos após a cura, sendo seu tratamento realizado nos serviços de referência ${ }^{(3)}$.

Dentre os fatores que podem contribuir para a ocorrência de recidivas, estão às características inerentes ao M.leprae, como por exemplo, torna-se pouco ativo e volta a se multiplicar após o término do tratamento (bacilos persistentes), como também fatores relacionados à cinética das drogas, irregularidade de tratamento pelo paciente ou alterações que possam levar à menor absorção ${ }^{(3)}$.

Não há consenso sobre os critérios estabelecidos para a confirmação diagnóstica de recidiva, pois há muitas variações nas manifestações clínicas, como o aparecimento de novas lesões, exacerbação de lesões antigas e/ou lesões de nervos com sinais clínicos e histopatológicos semelhantes com as formas ativas, aumento de índice baciloscópico (IB) $>2+$ em um ou mais sítios, lesões de nervos com ou sem neurites em casos de paucibacilar e comprovação diagnóstica por biópsia ${ }^{(4)}$.
O diagnóstico de recidiva em portadores da forma clínica paucibacilar é um desafio, pois a aparência das lesões cutâneas e as alterações histológicas são muitas vezes indistinguíveis da reação reversa tardia, que aparece normalmente durante a poliquimioterapia ou dentro de seis meses após finalização do tratamento em pacientes paucibacilares. Já a recidiva ocorre normalmente após um intervalo de cinco anos após o término da PQT ${ }^{(5)}$.

Quando se confirma uma recidiva - após exame clínico e baciloscópico -, a classificação do doente deve ser criteriosamente reexaminada para que se possa reiniciar o tratamento de PQT adequado $^{(1)}$.

A hanseníase, portanto, ainda é um importante problema de saúde pública no país. Dados epidemiológicos de Alagoas apontam que há uma gradativa diminuição do coeficiente de detecção de casos de hanseníase, pois no ano de 2013, o coeficiente de detecção de 10,93/100.000 habitantes, já em 2015 foi de 10,51/ 100.000 habitantes. Porém, considerando-se os parâmetros da OMS e do Ministério da Saúde, os dois períodos ainda exibiram alta taxa de detecção ${ }^{(6,7)}$.

A realização deste estudo é considerada de grande relevância, pois 
se pretendeu analisar o comportamento da recidiva de hanseníase em Alagoas, considerando a necessidade de conhecer a real situação, haja vista que, diferenciá-la da reação hansênica ainda é um desafio para os profissionais da saúde. Existe a possibilidade de classificação errônea destes casos, comprometendo a efetividade da assistência ao portador ou ex-portador de hanseníase.

Tendo essa problemática em vista, o presente estudo tem como objetivo analisar o comportamento dos casos de recidiva de hanseníase de residentes em Alagoas notificados e investigados, no período de 2001 a 2009. Além disso, o mesmo objetiva realizar o cruzamento dos dados da primeira notificação com a recidiva e avaliar as variáveis de pessoas, tempo e lugar dos casos.

\section{Método}

Trata-se de um estudo descritivo, retrospectivo com abordagem quantitativa dos portadores de hanseníase diagnosticado e notificados com recidiva, em Alagoas no período de 2001 a 2009. A população de estudo foi constituída de todos os casos confirmados de recidiva e foram notificados e investigados nos municípios e incluídos no banco de dados do Sistema de Informações de Agravos e Notificação (SINAN) gerenciados pela Diretoria de Vigilância Epidemiológica da Secretaria de Estado da Saúde de Alagoas.

Foram utilizados dados secundários coletados diretamente do banco de dados do SINAN NET, utilizando o arquivo hanseníase.bdf,os quais contém registros individualizados com todas as variáveis constantes das fichas individuais de notificação e de investigação de cada caso.

O estudo constou de uma análise das variáveis contidas no Banco de Dados do SINAN NET oriundas da ficha de notificação e investigação SINAN hanseníase (idade, sexo, forma clínica, local de residência e modo de entrada)

A análise das recidivas foi realizada tomando como base o período de 2001 a 2009. Foram utilizados os Software EPI INFO, EXCEL e SINAN NET. Ressalta-se que por se tratar de pesquisa com dados secundários, não foi necessário submeter o Projeto ao Comitê de Ética.

\section{Resultados}

Em Alagoas no período de 2001 a 2009 foram identificados no Sistema de Informações de Agravos e Notificação 
(SINAN), 103 casos com modo de entrada recidiva, desses 25 fichas foram excluídas por não terem sido localizadas no sistema de informação a primeira ficha de notificação com entrada como caso novo, uma excluída como recidiva no ano de 2010 após período de estudo, uma excluída por duplicidade.

Foi realizado o cruzamento de 76 fichas de notificação de hanseníase com modo de entrada de caso novo, no período estudado os dados denotam que 37 casos (49\%) realmente eram realmente recidivas e $39(51 \%)$ eram reações hansênicas, porque se enquadravam nos critérios preconizados pela Organização Mundial da Saúde (OMS) e do Ministério da Saúde (MS), que era o tempo mínimo após alta por cura (5 anos).

Segundo o gráfico1, os dados revelam que no primeiro ano avaliado foram registrados, 4 casos de recidivas de hanseníase e 1 reação hansênica, já no último ano avaliado foram 9 recidivas e 5 reações hansênicas.

\section{Gráfico 1: Número de Casos de Hanseníase, segundo Recidiva /Reação Hansênica em Alagoas}

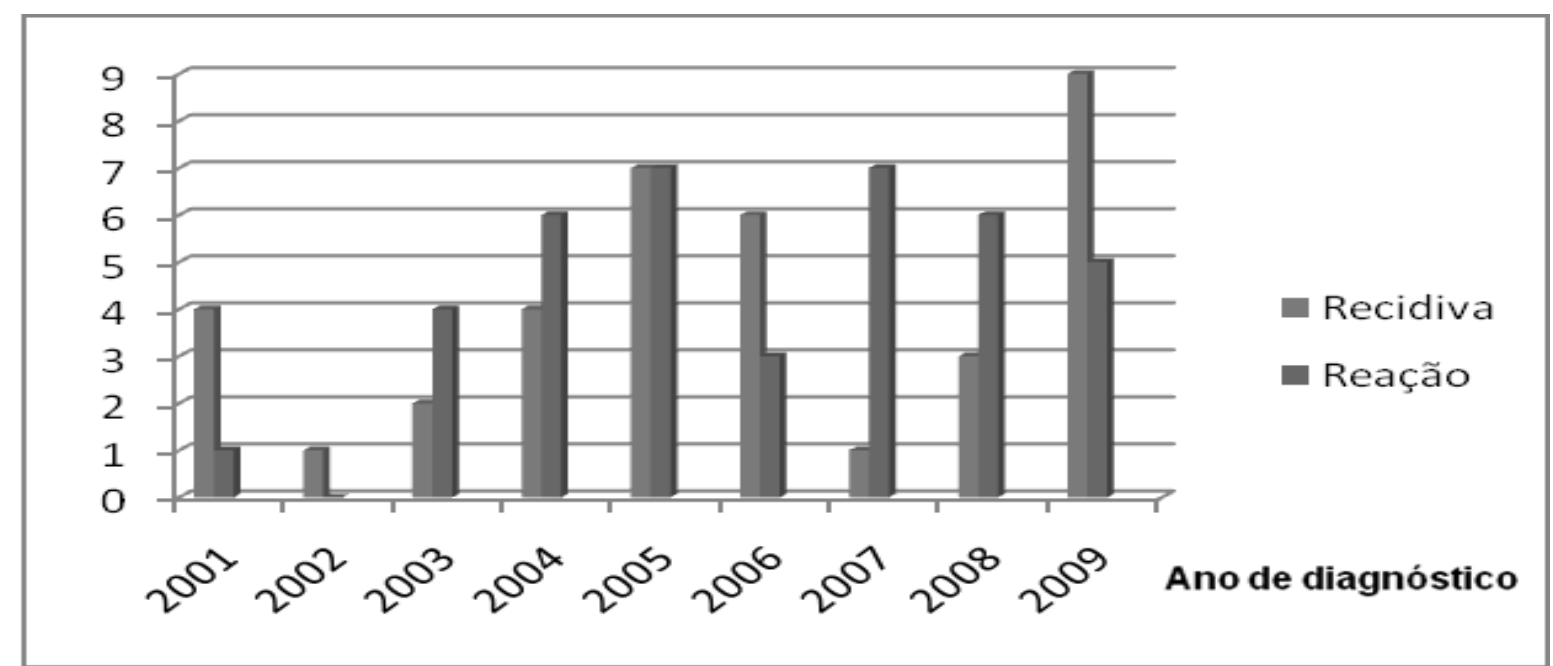

Fonte: SESAU/DIVEP/SINAN

A espacionalização dos casos evidencia a notificação de recidivas de hanseníase em 28 dos 102 municípios existentes em Alagoas. Desses, 14 era erro de diagnóstico, pois não tinham os cinco anos de alta por cura, conforme preconizado pelo MS, sendo classificados como reações hansênicas (Fig. 1). 


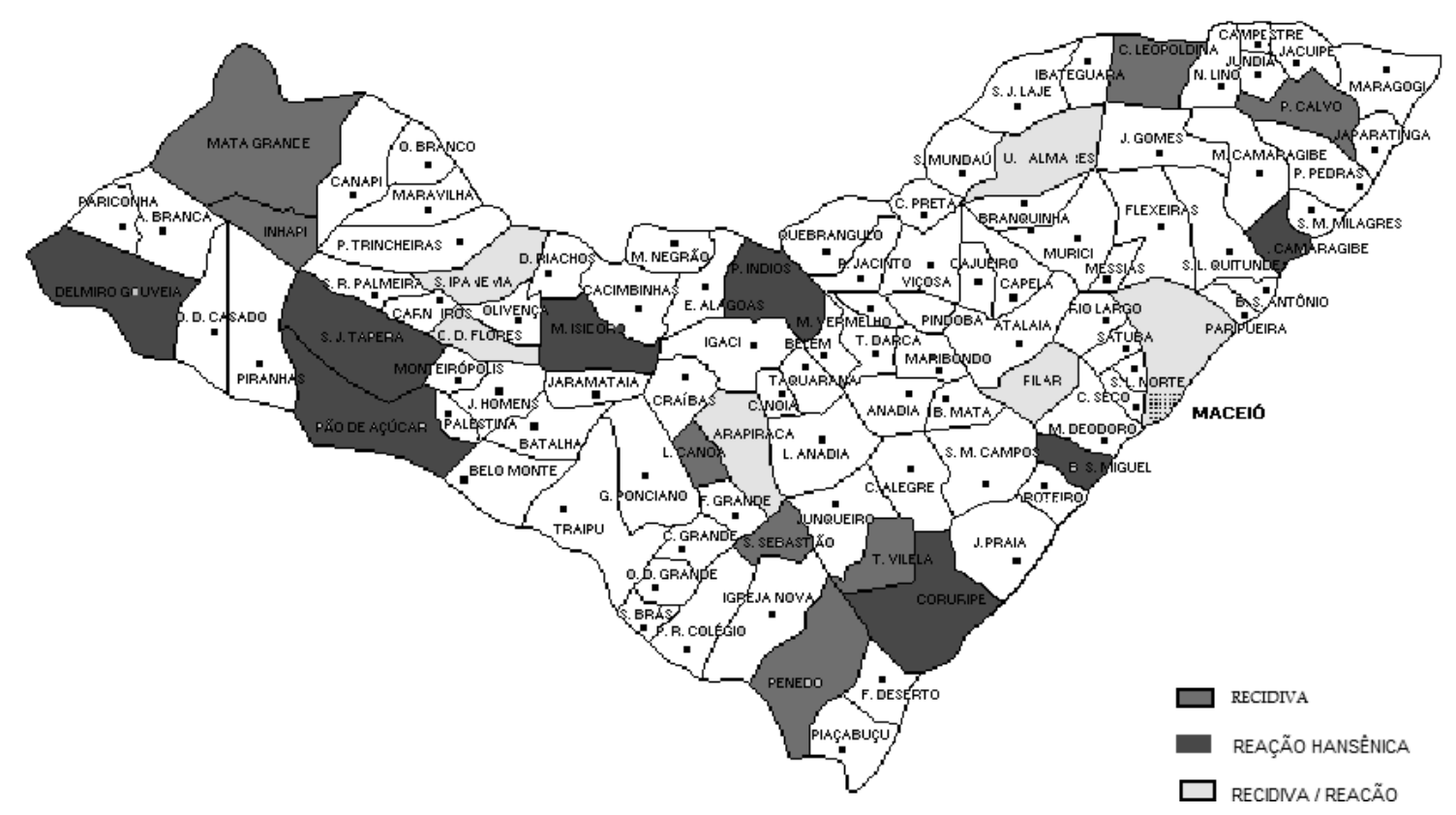

Fonte: SESAU/DIVEP/SINAN

Comparando os casos com modo de entrada como caso novo e recidiva a distribuição dos portadores de hanseníase quanto à forma clínica da doença no primeiro diagnóstico da hanseníase, 12 (32\%) apresentaram a forma indeterminada, $9 \quad(24 \%)$ tuberculóide, 7 (18\%) dimorfa, 7 (18\%) virchowiana, 2 (5\%) não foram classificadas e 1 (3\%) a informação estava em branco. Entre os doentes com recidiva de hanseníase, 8 (21\%) eram da forma indeterminada, $3 \quad(8 \%)$ tuberculóide, 10 (26\%) dimorfa, 11 (29\%) virchowiana, 4 (11\%) não foram classificadas e 2 (5\%) sem informação.
A tabela 1 mostra que a forma clínica que prevaleceu na $1^{\mathrm{a}}$ notificação foi à indeterminada que apresentou um índice de $32 \%$, na recidiva $31 \%$, na reação hansênica na $2^{\mathrm{a}}$ notificação foi a forma virchowiana com $29 \%$ e a dimorfa com $26 \%$.

Analisando os casos notificados de recidivas e reações hansênicas, segundo sexo, os dados revelam que o sexo masculino responde por $61 \%$ na recidiva e na reação hansênica foi o sexo feminino com maior índice (51\%).

O gráfico 2, revela que com relação à faixa etária, observou-se que o maior número de casos são os adultos/jovens (16 - 49 anos). 


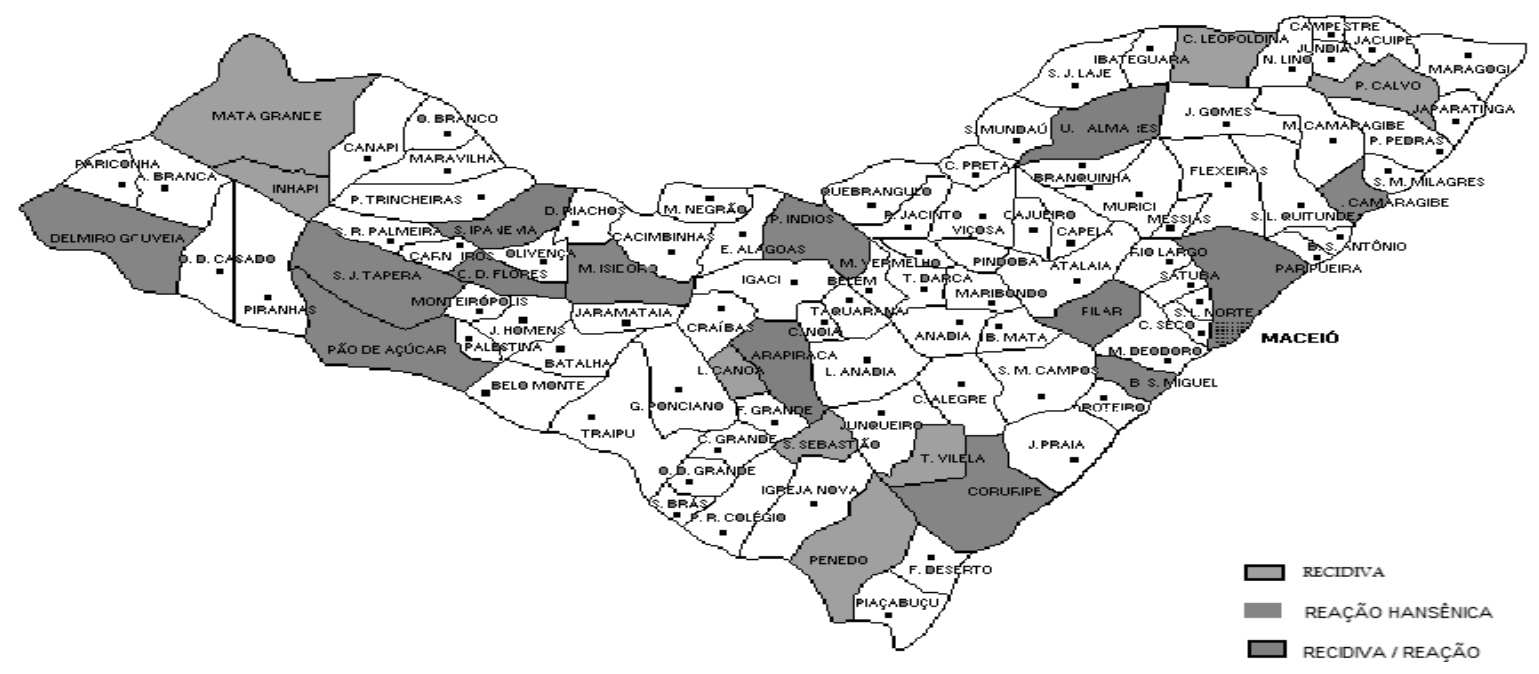

Tabela 1: Distribuição Forma Clínica, Recidivas e Notificações em Alagoas

\begin{tabular}{ccccccccc}
\hline & \multicolumn{2}{c}{ Recidiva } & \multicolumn{2}{c}{ Reação } & \multicolumn{2}{c}{ Recidiva } & \multicolumn{2}{c}{ Reação } \\
\cline { 2 - 9 } Forma Clínica & \multicolumn{2}{c}{$1^{\mathrm{a}}$} & \multicolumn{2}{c}{$1^{\mathrm{a}}$} & \multicolumn{2}{c}{$2^{\mathrm{a}}$} & \multicolumn{2}{c}{$2^{\mathrm{a}}$} \\
& \multicolumn{2}{c}{ Notificação } & \multicolumn{2}{c}{ Notificação } & Notificação & \multicolumn{2}{c}{ Notificação } \\
\cline { 2 - 9 } & $\mathrm{n}$ & $\%$ & $\mathrm{n}$ & $\%$ & $\mathrm{n}$ & $\%$ & $\mathrm{n}$ & $\%$ \\
\hline Indeterminada & 12 & $32 \%$ & 12 & $31 \%$ & 8 & $21 \%$ & 6 & $15 \%$ \\
Tuberculóide & 9 & $24 \%$ & 17 & $44 \%$ & 3 & $8 \%$ & 7 & $18 \%$ \\
Dimorfa & 7 & $18 \%$ & 3 & $8 \%$ & 10 & $26 \%$ & 10 & $26 \%$ \\
Virchowiana & 7 & $18 \%$ & 3 & $8 \%$ & 11 & $29 \%$ & 1 & $3 \%$ \\
Não Classificada & 2 & $5 \%$ & 3 & $8 \%$ & 4 & $11 \%$ & 11 & $28 \%$ \\
Em Branco & 1 & $3 \%$ & 1 & $3 \%$ & 2 & $5 \%$ & 3 & $8 \%$ \\
\hline
\end{tabular}

Fonte: SESAU/DIVEP/SINAN.

Gráfico 2 - Número de Casos de Hanseníase Segundo Modo de Entrada Recidiva E Reação em Alagoas

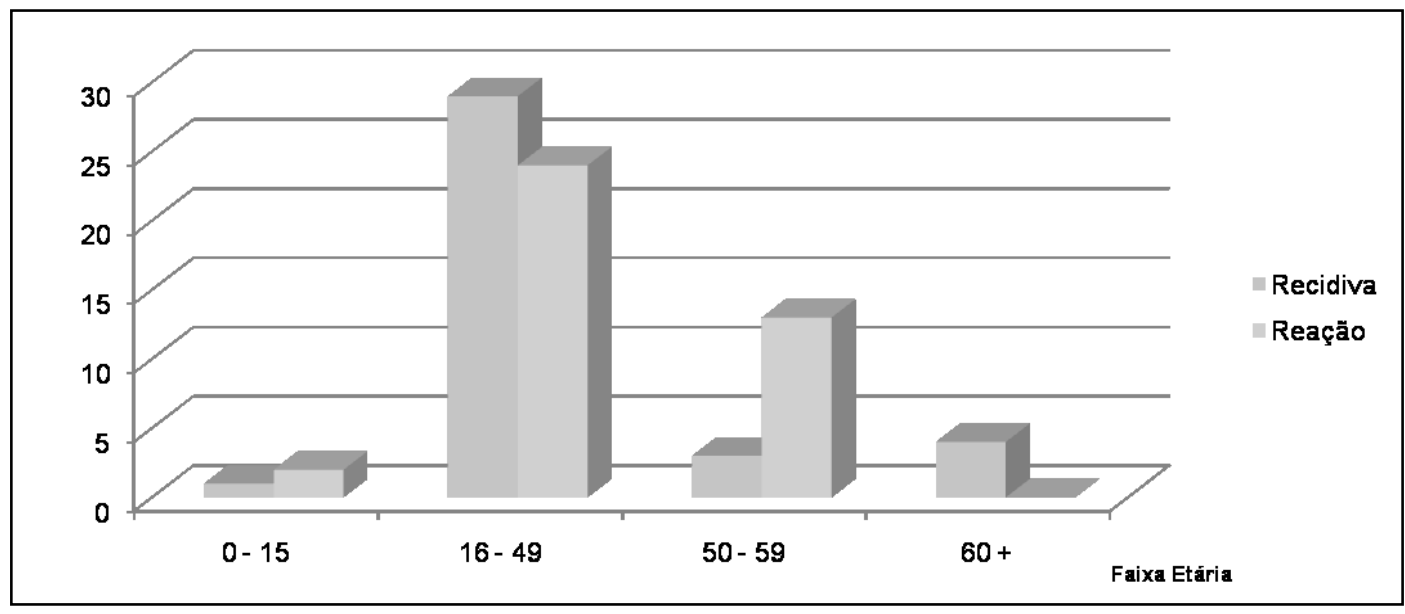

Fonte: SESAU/DIVEP/SINAN. 


\section{Discussão}

$\begin{array}{cccc}\text { O resultado deste } & \text { estudo } \\ \text { demonstrou que das } 76 \text { fichas }\end{array}$ analisadas 37 eram recidivas e 39 eram reações hansênicas. Sabe-se que a recidiva ocorre com o aparecimento de novos sinais e sintomas, após cinco anos em um indivíduo que recebeu alta por cura, já as reações podem ocorrer durante ou depois do tratamento $(8,10)$. Em um estudo transversal realizado no estado do Mato Grosso onde foram analisadas as entradas de recidivas em haseníase notificadas no período de 2004 a 2006 obteve um total de 323 registros com uma média de 107, 6 casos/ ano ${ }^{(9)}$. Outra pesquisa realizada no município de Teresina do estado do Piauí foi realizada um estudo de série no período de 2001 a 2008 obtidos pelo SINAN foi constatado 56 casos de recidivas $^{(8)}$.

Com relação aos estados reacionais também conhecidos como reações hansênicas é uma resposta do sistema imunológico da pessoa atingida pelo bacilo de Hansen. Sendo classificadas em tipo I ou reação reversa e tipo II ou eritema nodoso ${ }^{(10)}$. O primeiro tipo ocorre, principalmente, em pessoas diagnosticadas com a forma dimorfa e geralmente esses indivíduos estão no período do tratamento e de forma precoce, sendo que não exclui o aparecimento após o encerramento do tratamento, já o segundo tipo é mais frequente em pessoas que tiveram hanseníase virchowiana $(11,12)$. Um estudo retrospectivo realizado com 118 pacientes observou-se que 66 pessoas em estado reacional eram da forma virchoviana $^{(10)}$.

Em um estudo verificou que dentre os 104 estudados com recidiva de hanseníase, no primeiro diagnóstico, 37 pessoas apresentaram a forma indeterminada, 27 tuberculóide, 14 dimorfa e 26 virchowiana. Na recidiva da hanseníase, sete tiveram a forma indeterminada, 28 tuberculóide, 35 dimorfa e 34 virchowiana ${ }^{(13)}$.

Com relação à predominância dos casos de recidiva em relação ao sexo no Estado de Mato Grosso, no período de 2004 a 2006, a maioria $(71 \%, \mathrm{n}=229)$ dos doentes de recidiva era do sexo masculino ${ }^{(9)}$. Sendo que mulheres no período da gestação e lactação estão mais suscetíveis para recrudescimento ${ }^{(3)}$.

Em estudo retrospectivo de recidiva de hanseníase no Estado do Espírito Santo no período de 2000 a 2005, observou que dos 104 casos estudados a maior frequência foi entre 21 a 60 anos $^{(13)}$. Ao investigar 155 
indivíduos

diagnosticados

de

retratamento por recidiva de

hanseníase, verificou que as pessoas atingidas pela hanseníase estudadas, a maioria $(80,6 \%)$ tinha entre 15 e 65 $\operatorname{anos}^{(3)}$. Outros indicam que a faixa de concentração é em média $44 \operatorname{anos}^{(8)}$.

\section{Conclusão}

No estudo, após cruzamento de 76 fichas de notificação de hanseníase com modo de entrada de recidiva na segunda fichano período estudado, os dados revelaram que 37 casos (49\%), eram consideradas verdadeiras recidivas, segundo critérios OMS/MS, e 39 (51\%) de possíveis reações hansênicas, por não cumprir o número de anos de alta por cura (5 anos).

Observou-se que as entradas por recidiva se deram em $24(30 \%)$ em Unidades básicas e 55 (70\%) Unidades de Referência. Segundo dados de Alagoas no período selecionado as recidivas apresentaram um alto índice que corresponde a 5,4\% ao ano. Pode-se dizer que ainda existem dificuldades por parte dos profissionais de saúde das Unidades Básicas de Saúde e Referência em realizar esse diagnóstico de recidivas segundo parâmetros da OMS diferenciando das reações hansênicas. Outro achado identificado na pesquisa foi que não é uma prática corriqueira que os casos de suspeição de recidiva sejam encaminhados para unidade de referência para elucidação e definição dos casos.

O estudo aponta para a necessidade de capacitação e supervisão como educação permanente para os profissionais de saúde, que atuam nos municípios na atenção básica e unidades de referência para melhor identificação no manejo para recidiva $\mathrm{x}$ reação hansênica.

\section{Referências}

1. Ministério da Saúde (BR). Secretaria de Vigilância em Saúde. CoordenaçãoGeral de Desenvolvimento da Epidemiologia em Serviços. Guia de Vigilância em Saúde. Brasília: Ministério da Saúde; 2016.

2. Avelino AP, Pereirao AM, Ribeiro F, Castro JL, Mariana BA, Ramos NM. Perfil epidemiológico da hanseníase no período de 2009 a 2013 no município de Montes Claros (MG). Medicina (Minas Gerais) [periódico na Internet]. 2015 [citado 2017 abr. 23]; 13(3):1804. Disponível em: http://files.bvs. br/upload/S/1679-1010/2015/v13n3/a5 389.pdf3.

3. Ferreira SMB, Ignotti E, Gamba MA. Características clínicolaboratoriais no retratamento por recidiva em hanseníase. Epidemiologia (São Paulo) [periódico na Internet]. 2012 [citado 2016 nov. 10]; 15(3): 573-81. Disponível em: http://www .scielo.br/pdf/rbepid/v15n3/11.pdf. 
4. Pires CAA, Malcher CMSR, Júnior JMCA, Albuquerque TG, Corrêa IRS, Daxbacher ELR. Hanseníase em menores de 15 anos: a importância do exame de contato. Pediatria (São Paulo) [periódico na Internet]. 2012 [citado 2016 nov. 10]; 30(2): 292-5. Disponível em: http://www.scielo.br/p $\mathrm{df} / \mathrm{rpp} / \mathrm{v} 30 \mathrm{n} 2 / 22 . \mathrm{pdf}$.

5. Suzuki K, Akama T, Kawashima A, Yoshihara A, Yotsu RR, Ishi N. Current status of leprosy: epidemiology, basic science and clinical perspectives. Dermatology [internet newspaper]. 2012 [quoted 2016 nov. 10]; 39(2): 121-9. Available in: http://onlinelibrary.wiley.com/doi /10. 1111/j. 1346-8138.2011.01370.x/ pdf.

6. Ministério da Saúde (BR). Departamento de Informação e Informática do SUS. DATASUS. Informações de Saúde: Epidemiológicas e Morbidades. Brasília: Ministério da Saúde; 2013.

7. Ministério da Saúde (BR). Situação Epidemiológica da hanseníase - dados. Brasília: Ministério da Saúde; 2015.

8. Bona SH, Silva LOBV, Costa UA, Holanda AON, Campelo V. Recidivas de hanseníase em Centros de Referência de Teresina, Piauí, 20012008. Epidemiologia (Brasília) [periódico online]. 2015 [citado 2017 abr. 23]; 24(4): 731-38. Disponível em: http://scielo.iec.pa.gov.br/scielo. php?s cript $=$ sci_arttext\&pid=S1679-4974201 5000400015.

9. Ferreira SMB, Ignotti E, Senigalia LM, Silva DRX, Gamba MA. Recidivas de casos e hanseníase de Mato Grosso. Saúde Pública (Mato Grosso) [periódico online]. 2010 [citado 2017 abr. 23];44(4):650-7. Disponível em: http://www.scielo. br/pdf/rsp/v44n4/08.pdf.
10. Souza LWF. Reações hansênicas em pacientes em alta por cura pela poliquimioterapia. Medicina (Minas Gerais) [periódico na Internet]. 2010 [citado 2017 abr. 23]; 43(6): 737-39. Disponível em: http://www.scielo.br/ $\mathrm{pdf} / \mathrm{rsbmt} / \mathrm{v} 43 \mathrm{n} 6 / 29$.pdf.

11. Andrade ARC, Nery JAC. Hanseníase avanços e desafios. Brasília: NESPROM; 2014; 1: 18690.

12. Foss NT. Episódios Reacionais na Hanseníase. Medicina (Ribeirão Preto) [periódico na Internet]. 2003 [citado 2017 abr. 25]; 36: 453-9. Disponível em: http://revista.fmrp.usp.br/2003/36n 2e4/37episodios_reacionais_hansenias e.pdf.

13. Diniz LM, Moreira MV, Puppin MA, Oliveira MLWDR. Estudo retrospectivo de recidiva da hanseníase no Estado do Espírito Santo. Rev. Revista da Sociedade Brasileira de Medicina Tropical [periódico na Internet]. 2009 [citado 2016 nov. 10]; 42(4): 420-4. Disponível em: http://www.scielo.br/pdf/rsbmt/v42n4/ a12v42n4.pdf. 